\title{
The future of cancer therapy
}

\section{American Association of Cancer Research meeting notes, Washington, April 2006}

$\mathrm{T}$ he 97th Annual Meeting of the Association for American Cancer Research (AACR), held in Washington, DC from April 1st to 5th, 2006, was attended by over 17,000 people who submitted more than 6,100 abstracts. This annual conference is the world's largest in the cancer research field. the nearly 100 symposia and numerous forums, educational sessions, and methods workshops covered diverse topics from tea catechins to in vivo imaging to cancer stem cells.

The dominant theme of the week, novel and promising advances in the treatment of cancer, was described in the Opening Plenary Session on Looking into the Future of Cancer Therapy. Bert Vogelstein, an expert in the field of cancer genetics, opened the session with a discussion of how to best help patients by implementing our knowledge of cancer as a genetic disease. Vogelstein suggested that research should be focused on developing genebased cancer screening and diagnosis strategies, allowing clinicians to identify high-risk patients and early-stage cancers. Additionally, targeted therapies that ameliorate genetic abnormalities present in cancers should be further investigated, despite many difficulties such as resistance due to the inherent genetic instability of tumour cells. By combining gene-based diagnostics and therapies that target genetic mutations in cancer patients, Vogelstein believes that cancer suffering could be reduced within the foreseeable future.

In addition to the future of cancer treatment, another prevailing trend within this meeting was cancer prevention. José Baselga, a leader in the field of breast cancer clinical research, chaired the first annual Clinical Plenary Session on Breakthroughs in Clinical Research. Four late-stage clinical trial results were revealed, including two independent trials that examined the chemopreventive effect of celecoxib on adenoma growth. The Adenoma Prevention with Celecoxib Study examined twice daily dosing of celecoxib. The Colorectal Sporadic Adenomatous Polyps with Celecoxib Trial studied once daily dosing. Both trials used year-one and year-three colonoscopies as endpoints and, although both showed decreased adenoma incidence in the treatment arms of the studies, patients taking celecoxib also demonstrated increased cardiovascular events. As a result, there was some debate at the end of the session over the best way to administer celecoxib to reduce adenoma incidence while minimizing patient risk.

Another session that resulted in animated discussion was regarding vitamin $\mathrm{D}$ and cancer risk. There is growing evidence that high vitamin D leads to decreased risk of breast and colorectal cancer and perhaps other types of malignancies. However, the majority of the population, especially in Northern latitudes, are vitamin D deficient due to both weather-limited sun exposure and the daily use of sunscreen. As a result, the discussion centered around the best way to obtain a daily dose of vitamin D, including dietary change, food fortification, supplementation, and sunlight exposure. Daily sunscreen-free exposure to the sun was decidedly the most controversial of the methods discussed.

A final key theme of this meeting was tumour biology, especially the tumour microenvironment, inflammation, and the metastatic process. Various sessions and posters emphasized the importance of chemokines in the dissemination and growth of secondary tumours as well as the involvement of inflammatory mediators in cancer development and progression. Frances Balkwill, one of the first researchers to describe a link between chemokine receptor expression and tumour spread, chaired an Educational Session on Chemokines as Mediators of Invasion and Metastasis. This session described the important role of chemokines, not only in invasion and metastasis, but also in tumour inflammation and angiogenesis. 


\section{E. Lowthers}

The Annual Meeting of the AACR was a very comprehensive meeting that outlined findings from a broad range of topics within the cancer research field. Abstracts presented at the meeting are now freely available at www.aacr.org. Those that garnered media attention, such as the finding that there is no link between aspartame intake and cancer incidence, are also highlighted on the AACR website. The 98th Annual Meeting will be held April 14th to 18th, 2007, in Los Angeles, CA and promises to be another excellent venue for the dissemination of ideas and findings of cancer researchers from around the world.

\section{Erica L. Lowthers \\ Department of Pharmacology, Dalhousie University}

\section{The function of lipin in obesity and lipodystrophy}

\section{Experimental Biology meeting notes, San Francisco, April 2006}

$\mathrm{E}$ xperimental Biology is the lead scientific conference for the American Association of Anatomists, the American Physiological Society, the American Society for Biochemistry and Molecular Biology, the American Society for Investigative Pathology, the American Society for Nutrition, and the American Society for Pharmacology and Experimental Therapeutics. The conference was attended by $\sim 15,000$ scientists, physicians, and trainees. Thematic symposia and poster presentations were presented over four days. With such a large meeting it is impossible to attend every event, especially as many overlap. Of the symposia I attended, one of the most important presentations was work out of Rutgers University where the function of the lipin gene was determined. Lipin is one of only a handful of genes that can adjust body fat up or down, depending on its expression level. Increased levels of lipin result in heightened insulin sensitivity as well as obesity. Decreasing lipin levels results in insulin resistance and lipodystrophy. Lipin is thus considered to be a very good target for the development of drugs for the treatment of both obesity and lipodystrophy ${ }^{1}$.

At the Experimental Biology conference, lipin was recognized as the missing gene in the biochemical pathway that converts fatty acids into triacylglycerol. New research presented at the conference, identified the function of the lipin protein as an enzyme called phosphatidic acid phosphatase (PAP), which converts a lipid (fat) metabolite called phosphatidic acid into diacylglycerol ${ }^{2}$. Subsequent to this enzymatic conversion by lipin, a fatty acid is then added to diacylglycerol to produce an intact triacylglycerol fat molecule. Excessive PAP leads to an increase in triacylglycerols and consequently fat deposition. Identifying the mechanistic basis of lipin function now enables direct screening of small molecules for inhibition or activation of lipin encoded PAP activity. With this discovery, the hope of new therapies for obesity and lipodystrophy are closer at hand.

\section{Christopher R. McMaster \\ Departments of Pediatrics and Biochemistry \& Molecular Biology, Faculty of Medicine, Dalhousie University}

\section{References}

1. Phan J, Reue K. (2005) Lipin, a lipodystrophy andoobesity gene. Cell Metab. 1, 73-83.

2. Han GS, Wu WI, and Carman GM (2006) The Saccharomyces cerevisiae lipin homolog is a $\mathrm{Mg} 2+-$ dependent phosphatidate phosphatase enzyme. J. Biol. Chem. 281, 9210-9218. 